\title{
Observatorios económicos y de emprendimiento desde la academia
}

Mario Roberto Acevedo Amaya*, Jesús Argueta Moreno*, Cesar Humberto Ortega Jiménez*

\section{RESUMEN}

A nivel mundial los Observatorios Económico y de Emprendimiento (OEE) buscan incrementar las potencialidades de una nación, y en especial cuando son realizados desde la academia, buscan potencializar esas capacidades que tienen las universidades para responder de manera oportuna a las necesidades de conocimiento científico-tecnológico del sector productivo de un país.

En este sentido, las actividades de los OEE desde la academia deberán ser un instrumento estratégico fundamental que articule el Sistema Nacional de Monitoreo y Evaluación de los distintos sectores económicos, así como las cuentas nacionales, mediante la identificación de las necesidades de conocimiento del sector productivo y de la producción de conocimientos relevantes en la universidad. Sumado a ello, la adopción de mecanismos adecuados de investigación, desarrollo e innovación que permitan una vinculación, aprendizaje y difusión.

Los OEE desde las academias constituyen el desarrollo pionero en la investigación oportuna de la realidad económica y empresarial de nuestro país, con la finalidad de adoptar los correctivos necesarios en la economía, a través de los "tanques de pensamiento" como instrumento de política universitaria para apoyar en etapas tempranas, con un sentido prospectivo, o en etapas posteriores con un sentido correctivo. A su vez, los OEE colaboraran con las actividades de importancia social, económica y empresarial que pudieran requerir la acción oportuna de la universidad en conjunto con los distintos actores a fin de crear conciencia critica y toma de decisiones efectivas.

Palabras clave: Observatorios económicos, emprendimiento, indicadores económicos, tanques de pensamiento.

"Universidad Nacional Autónoma de Honduras. Facultad de Ciencias Económicas. Instituto de Investigaciones Económicas y Sociales.macevedo@iies-unah.org; jargueta@iies-unah.org; cortega@iies-unah.org 


\section{ABSTRACT}

The Economic and Entrepreneurship Observatory (OEE) seeks to increase the potentials of country, in special when are from the academy. By quickly detecting and responding to the various needs of scientific and technological knowledge among all the productive sectors (manufacturing of products or services). In this way, the activities executed by the OEE pursuit the development of the fundamental strategic tools that shall mediate the articulation of the National Monitoring and Evaluation of both, the business sector and the national accounts.

The identification of the potential claims of knowledge, related to the productive sector and the generation of relevant investigations at the university, joined to the adoption of learning and dissemination mechanisms, will eventually buildup a pioneer project, that will upgrade the business reality of our country. The creation of the "Think Tanks" as university policy tool, to support the working activities of potential social, economic and businesses, may require timely action of the universities personnel in conjunction with the various stakeholders in decisionmaking of our countries finance sectors.

Key words: Economic Observatory, Entrepreneurship, Economic Indicators, Think Tank. 


\section{INTRODUCCIÓN}

La presente investigación es una adaptación derivada de la ponencia realizada ante el congreso LACCEI 2012 en Panamá. Por lo tanto, se pretende con ello fortalecer los conocimientos expuestos en el congreso antes mencionado, a fin de dar secuencia a la investigación en el marco del apoyo que la Dirección de Investigación Científica da a los docentes investigadores de la UNAH.

Los centros educativos a nivel mundial son considerados como espacios de gran importancia para la generación de conocimiento a partir de procesos educativos y formativos innovadores que coadyuvan a la consecución de los objetivos de una nación. Las actividades desempeñadas por los centros de Educación Superior en el mundo revisten un carácter científico sustentado en la generación de InvestigaciónDesarrollo-Innovación permitiendo a los países más desarrollados recibir aporte en materia de políticas de estado y de gobierno, surgidas a partir de la conciencia crítica desarrollada desde la academia. Es por ello que, los OEE desde la academia, tienen como rol, ser un espacio para la investigación, reflexión y difusión de la actualidad económica y empresarial.

Se colaborará, a través de alianzas estratégicas con el sector público y privado, a afrontar los retos en materia económica nacionales e internacionales que se presenten. LOS OEE involucran la parte teórica por medio de la investigación y búsqueda minuciosa en las literaturas económicas, así como publicaciones y medios electrónicos que proporcionan información actualizada en tiempo real de los mercados a nivel local y mundial. Asimismo, los OEE fomenta la participación activa de sus integrantes en conferencias, publicaciones, foros y espacios para debates entre la academia y gestión pública, atrayendo a la cooperación externa e interna y los distintos sectores de la sociedad Hondureña.

Los observatorios desde la academia y en especial los OEE proveen herramientas de medición que se constituirán en el soporte para avanzar en la generación de un desarrollo sostenible en el sector económico que permita establecer ventajas competitivas e identificar oportunidades y estrategias de fortalecimiento y desarrollo a corto, mediano y largo plazo para el país. Por otra parte, el desarrollo del OEE desde la academia dará seguimiento al comportamiento de los indicadores económicos y empresariales del país, la cual a través de consultorías, estudios y puesta en práctica de teorías desarrolladas, podrá facilitar la toma de decisiones a autoridades, empresarios, responsables políticos, empresariales y a la sociedad en su conjunto. 
Por otra parte, el componente empírico de los OEE desde la academia, desarrolla una base de datos cuyo principal contenido es la información de enlace, rubro, segmento de mercado, posicionamiento, competitividad, rendimiento, etc. de empresas de bienes y servicios, para potenciar el flujo de información y conocimiento de la Universidad Nacional Autónoma de Honduras (UNAH) hacia el sector productivo, todo ello mediante la puesta en marcha de una red solida de información entre la UNAH y todas las instituciones con datos socioeconómicos, empresariales y estadísticos relevantes en el país.

Es por ello que la información recibida por el OEE combinará la teoría con la continua investigación cuantitativa y cualitativa para estudiar de forma profunda los cambios en la economía, a su vez, creará una conciencia crítica sobre la situación actual que permita diseñar e implementar modelos y políticas en común, para alcanzar beneficios colectivos. La retroalimentación que emane del OEE, transfiere a los estudiantes, las empresas y a la sociedad en general, un valor agregado en la formación académica como factor clave en la competitividad. Cada uno de los resultados del OEE serán validados y confiabilizados por la pluridisciplinariedad y experiencia previa de los componentes del grupo de investigación del IIES-UNAH, la cual avalan la gestión efectiva, eficaz y eficiente que deberá tener.

Ante tal situación, los análisis deben ser mucho más dinámicos que los que actualmente se desarrollan, conduciendo a la adopción de nuevas medidas y políticas económicas mucho más efectivas y eficientes. Desde esta perspectiva, se trata que el OEE provea insumos informativos sobre el desempeño empresarial y económico, así como la eficiencia de las políticas económicas y sociales implementadas, a fin de establecer y adoptar los correctivos necesarios, que permitan prevenir y retomar el rumbo en la conducción de la gestión pública, privada y la propia actividad económica del país, por sendas menos erróneas.

En este sentido, el Observatorio Económico y de Emprendimiento representa una gran oportunidad para la academia, debido a que es desde el centro de estudios donde se desarrollan los aportes de información acerca de la situación económica actual a todos los niveles, la cual son temas sensitivos y de gran relevancia para todo el conglomerado hondureño. Sumado a ello, desde el punto de vista jurídico institucional, la legislación universitaria de la UNAH, faculta al Instituto de Investigaciones Económicas y Sociales (IIES) para llevar adelante la investigación como uno de los pilares básicos en que descansa el quehacer universitario y una vía de articulación entre la docencia y la vinculación institucional con la sociedad. La oportunidad del OEE radica en la siguiente problemática plasmada en dos grandes interrogantes: ¿En qué medida la información (indicadores económicos y 
empresariales) provista por las instituciones especializadas del Estado, sirven para prever o predecir futuras crisis o calificar los ciclos económicos a corto plazo? Con todos los insumos informativos que nos proporcionan la autoridad monetaria del país y los períodos de tiempo que utilizan para facilitar dicha información, ¿serán suficientes para adoptar las precauciones en el ámbito económico requeridas?

Si bien es cierto, los indicadores económicos y sociales provistos por las instituciones públicas, se producen sobre la base de metodologías apropiadas y refrendadas por convenciones internacionales y constituyen, los datos oficiales acerca del desempeño y funcionamiento de la sociedad hondureña en su conjunto, no obstante, generan cierta resistencia de algunos grupos de presión. De manera que la provisión de información de esta naturaleza, sobre todo indicadores de muy corto plazo, no sólo deben contribuir para que instituciones como la UNAH articulen la docencia con la investigación y la vinculación institucional con la sociedad, propiciando una estrecha relación entre la academia y la práctica social, como laboratorio para una conciencia crítica, que se incube en el verdadero conocimiento de la realidad económica nacional.

Es por ello que la información que se origine a partir del OEE, aporta de forma constante su concurso para legitimar el trabajo realizado por aquellas instituciones públicas encargadas de la producción de los datos económicos estadísticos. En la misma perspectiva de complementariedad, debe señalarse que los espacios de producción de información especializada, se habrán de acortar con el observatorio, dado que la generación de datos se hará con periodicidad constante, lo que de esta forma permite crear los elementos de análisis y previsión, que permitan reducir los sesgos y retardos de la política económica; pero que fundamentalmente, servirán para facilitar la toma de decisiones oportunas y adecuadas, a fin de propiciar una mejor conducción de la actividad económica en el país.

\section{OBJETIVOS Y HIPÓTESIS}

La hipótesis de la presente investigación radica en que el Observatorio Económico y de Emprendimiento (OEE) desde la academia, deberá ser un instrumento estratégico fundamental que articule el Sistema Nacional de Monitoreo y Evaluación de la Economía del país, mediante la identificación de las demandas potenciales de conocimiento del sector productivo y de la producción de conocimiento relevantes en la universidad, así como la adopción de mecanismos adecuados de vinculación, aprendizaje y difusión.

El objetivo concreto del OEE consiste en producir información científica de forma 
oportuna acerca de la realidad económica y empresarial del país, la cual se constituiría como un insumo básico para realizar estudios e investigaciones en tiempo real y a futuro, con el propósito de adoptar los correctivos necesarios que deriven en un mejor funcionamiento tanto de la economía nacional como del sector empresarial, así como de la sociedad en su conjunto.

\section{METODOLOGÍA}

La metodología empleada en la presente investigación requiere de varios elementos debido a que el tema es amplio con un marcado carácter multidisciplinar. A su vez, requiere de actividades de diversas naturalezas influyendo de forma directa en la metodología de trabajo, que también presenta un carácter multivariado.

Por lo tanto, a continuación detallamos las diferentes actividades a desarrollar y la metodología a seguir:

1. Estudio a nivel local e internacional y de forma permanente del funcionamiento tanto operativo, investigativo y académico de los Observatorios Económicos y de Emprendimiento.

2. Recolección de Bibliografía y casos de éxitos en el mundo acerca de los Observatorios Económicos y de Emprendimiento.

3. Selección de la masa critica experta interna y externa para la dirección Observatorio.

4. Selección de la masa critica estudiantil de pregrado para integrar el Observatorio.

5. Selección de la masa critica de Posgrado para colaboración con el Observatorio.

6. Desarrollo de Indicadores Económicos para seguimiento, evaluación, análisis y salidas de investigación.

7. Desarrollo de cuestionarios para la medición de los indicadores micro y macro económicos.

8. Selección y recolección de datos de la muestra.

9. Análisis estadístico de los Datos.

10. Selección de docentes y facultades colaboradoras para la recolección, análisis y difusión de la información.

11. Difusión de Resultados y capacitaciones al respecto. 


\section{LA ACADEMIA Y LOS OBSERVATORIOS ECONÓMICOS Y DE EMPRENDIMIENTO}

Hoy en día, resulta primordial vincular los observatorios a la academia, ya que es en la etapa de formación de los estudiantes donde se gestan los futuros empleadores del estado que tendrán en sus manos la toma de decisiones transcendentales para el país. En este sentido, los Observatorios Económicos y de Emprendimiento desde la academia resultan ser un reto importante de afrontar por cada uno de los entes encargados de impartir el conocimiento a niveles de Educación Superior. Un país sin estadísticas y son controles y más aún, sin expertos generando conciencia critica en todos los niveles educativos, sólo da pie a sucumbir en el subdesarrollo, en cambio un Estado con estadísticas puntuales originadas a partir de conocimiento experto da lugar al desarrollo paulatino de su economía y país. Si bien es cierto invertir en educación resulta erogar grandes sumas, sin embargo, no invertir en educación resulta ser mucho más caro, y más aun si el país se encuentra en vías de desarrollo. Por lo tanto, la creación de los OEE desde la academia deberá tener como función; diseñar un proceso de investigación activa y sistemática, cuya principal actividad será identificar, analizar, interpretar y difundir información oportuna de valor estratégico sobre ciencia, economía y administración que oriente para la toma de decisiones estratégicas en el país. El monitoreo y evaluación realizado por el OEE da como salida una alerta temprana en el sentido prospectivo o en etapas posteriores con un sentido correctivo, acerca de desarrollos científicos, socioeconómicos y empresariales.

Ante ello, el OEE tienen como parte de sus actividades principales la creación de una red de expertos que involucren la investigación con la innovación y el desarrollo, estos expertos estarán compuestos por docentes universitarios y demás actores involucrados de forma directa con los aspectos económicos del país. Los expertos contaran con el apoyo de los estudiantes de pregrado y posgrado de la UNAH a fin de crear un sistema de colaboración en dos vías, la primera consistirá en la experiencia y los conocimientos en materia de investigación que transmitan los expertos a los estudiantes, y la segunda consistirá en el apoyo en la recolección de datos, creación de conciencia critica, estudios de campo y reflexión, entre otras, que realizarán los estudiantes para alimentar de forma constante las bases de datos en ámbitos sociales, económicos, empresariales y de la información. La conjunción de la red de expertos, estudiantes y la colaboración del grupo coordinador proporcionará y suministrarán la información al sitio WEB del OEE para la generación de nuevas salidas de investigaciones, tales como: 1. Comunicación científica y profesionalizante, 2. Paneles de expertos, 3. Conferencias, 4. Ponencias, 5. Entrevistas, 6. Debates 
El OEE tendrá entre sus funciones la creación de un sistema que articule: 1. Expertos, 2. Estudiantes, 3. Docentes, 4. Equipo de investigadores del Instituto de Investigaciones Económicas y Sociales (IIES-UNAH).

Los antes citados deberán basarse en las líneas de investigación de la Facultad de Ciencias Económicas para que a través de la conjunción de los mismos, mediante los procesos de investigación, innovación y desarrollo, pueda evaluar de forma continua los aconteceres en el campo económico que se están suscitando en el ámbito nacional, así como internacional. A continuación se presenta de forma general el proceso del OEE:

Figura 1. Proceso del Observatorio Económico Empresarial, Fuente: IIES-UNAH 2011

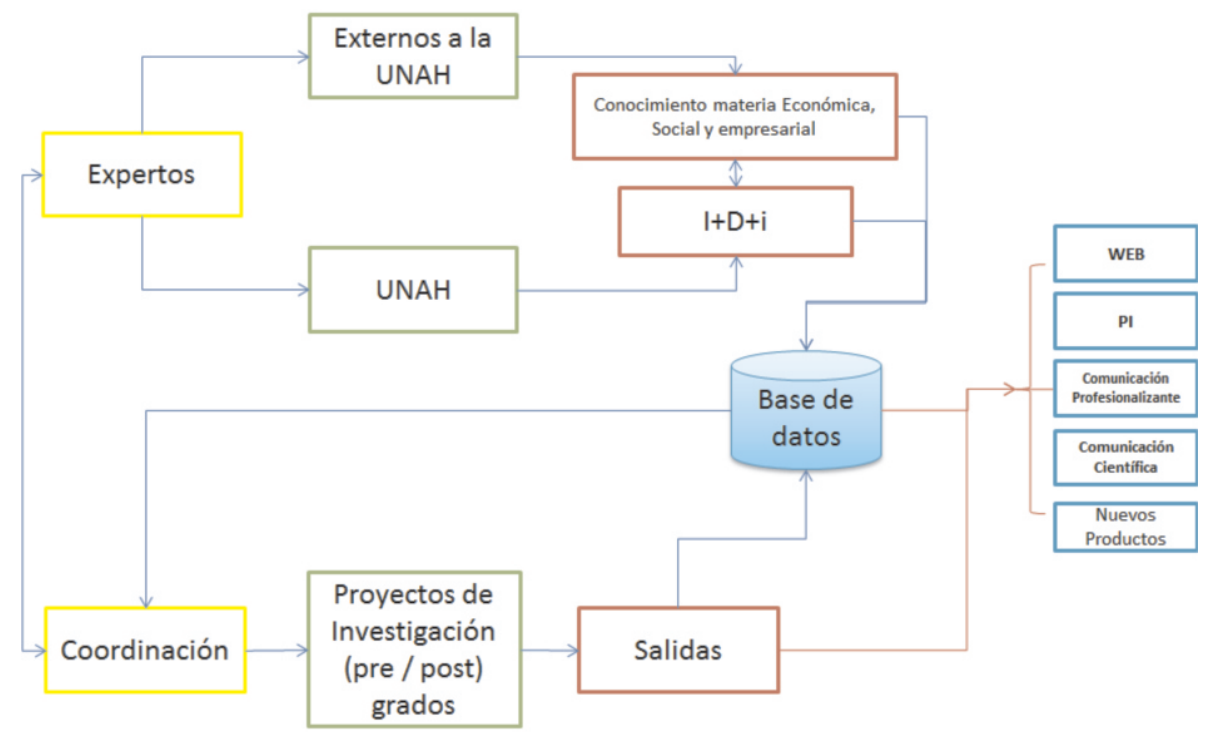

\section{LOS OBSERVATORIOS Y LOS EMPRENDIMIENTOS}

El Emprendimiento proveniente del francés Entrepreneur, cuyo significado es ser pionero, ha estado presente a lo largo de la historia de la humanidad, debido a que es inherente a esta. Es por ello que, en el afán de la importancia que reviste este novedoso pero antiguo concepto, el OEE realiza todas las acciones que le competan a fin de estimular en la academia el desarrollo del emprendimiento. Por 
otra parte, el emprendimiento se define como la actitud y aptitud de la persona 0 grupo de personas para emprender nuevos proyectos, nuevas ideas o concebir nuevos retos, permitiéndole avanzar e ir más allá de donde actualmente ha llegado. En otras palabras, es la insatisfacción que vive en el ser humano que lo motiva a seguir adelante para alcanzar nuevos logros en todos los ámbitos de su vida de forma constante e innovadora.

El emprendimiento reviste un carácter importante y fundamental hoy en día, ante los altos niveles de desempleo que se viven en américa latina y en especial los países de la región centroamericana, lo que generan en las personas la necesidad de agenciarse de sus propios recursos, a través de las iniciativas de formar negocios propios, ideas o modelos teóricos, que les permitan pasar de ser empleados a empleadores, en términos sencillos, recurren al emprendimiento como medio de escape a la problemática económica existente(Drucker, 1985).

No obstante, en la mayor parte de los casos, las iniciativas se ven diluidas de forma espontánea ante la ausencia de un ente que respalde y acompañe en él estudio, desarrollo y ejecución para llevar a la realidad esa idea de emprendimiento. Ante dicha problemática, el OEE en su área de emprendimiento permite al estudiante monitorear los proyectos que surjan de las incubadoras de ciencia y emprendimiento. El monitoreo admite contextualizar su idea a través de los estudios de investigación científica, viabilidad y factibilidad, para ser insertado al sector productivo, económico y empresarial. Dicho monitoreo del comportamiento de las empresas, investigaciones e emprendimiento le dan la oportunidad de pasar de investigadores emprendedores a generador de mano de obra.

Desde el punto de vista de enfoque, muchos emprendedores se basan en el enfoque de ver oportunidades de negocio en ideas que permitan satisfacer las necesidades de otros. Sin embargo, los emprendedores generalmente no se enfocan en los problemas o dificultades porque encuentran un estímulo en resolver cosas y en la mayor parte de las ocasiones encontrar un modelo de negocio en ello, no obstante para no fracasar se requiere de enfoques que les permitan una definición de prioridades. En este sentido, el OEE se consolida como el centro donde los proyectos derivados de las incubadoras encuentran un lugar de control, monitoreo, mejora y visión de cada uno de los cambios y evoluciones que presente el mismo.

Lo que permitirá eliminar errores comunes tales como muchas ideas, pero poca ejecución, falta de enfoques, conocimientos de delegación, entre otros. EI OEE en la actualidad se encarga de enseñar al emprendedor a tomar decisiones trascendentales, enfocarlo en un sólo proyecto y lograr que éste sea el mejor en ello, 
inculcarle a tener una visión que permita estar claro de dónde viene, por donde va y hasta donde quiere llegar. Cada uno de los aspectos antes expuestos, son las temáticas de soporte que desarrollará el OEE para dar un apoyo integral a sus emprendedores.

Figura 2. Proceso de la vinculación del Emprendimiento con el OEE, Fuente: IIES-UNAH 2011

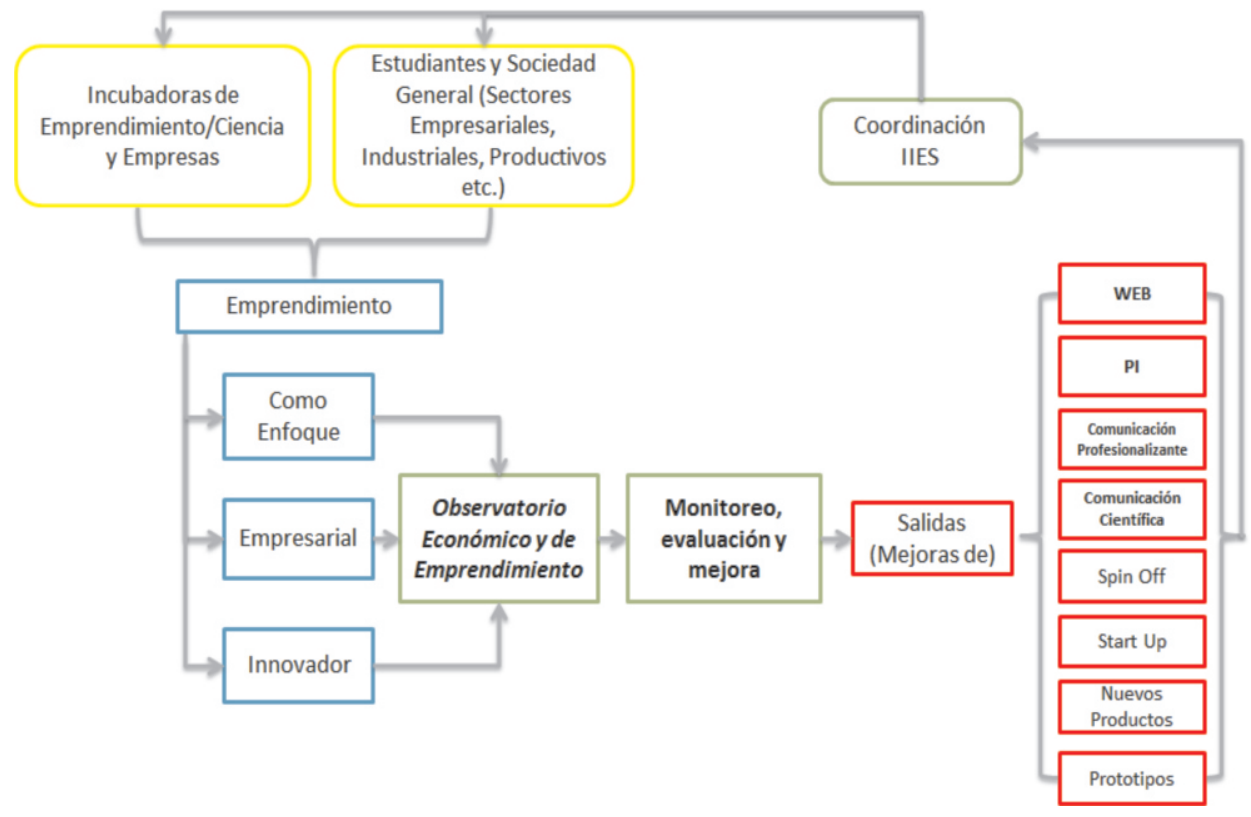

\section{SISTEMATIZACIÓN DEL OBSERVATORIO ECONÓMICO Y DE EMPRENDIMENTO}

El Observatorio Económico y de Emprendimiento desde las academias deberán estar sistematizados y enriquecidos mediante las incubadoras de ciencia y emprendimiento, así como los proyectos externos que busquen en el OEE el lugar idóneo para su desarrollo y el grupo de expertos a lo interno y externo de la Universidad que darán mayor sostenibilidad científica a los resultados del Observatorio.

Representado gráficamente de la siguiente forma: 
Figura 3. Proceso de las OEE sistematizado, Fuente: IIES-UNAH 2011

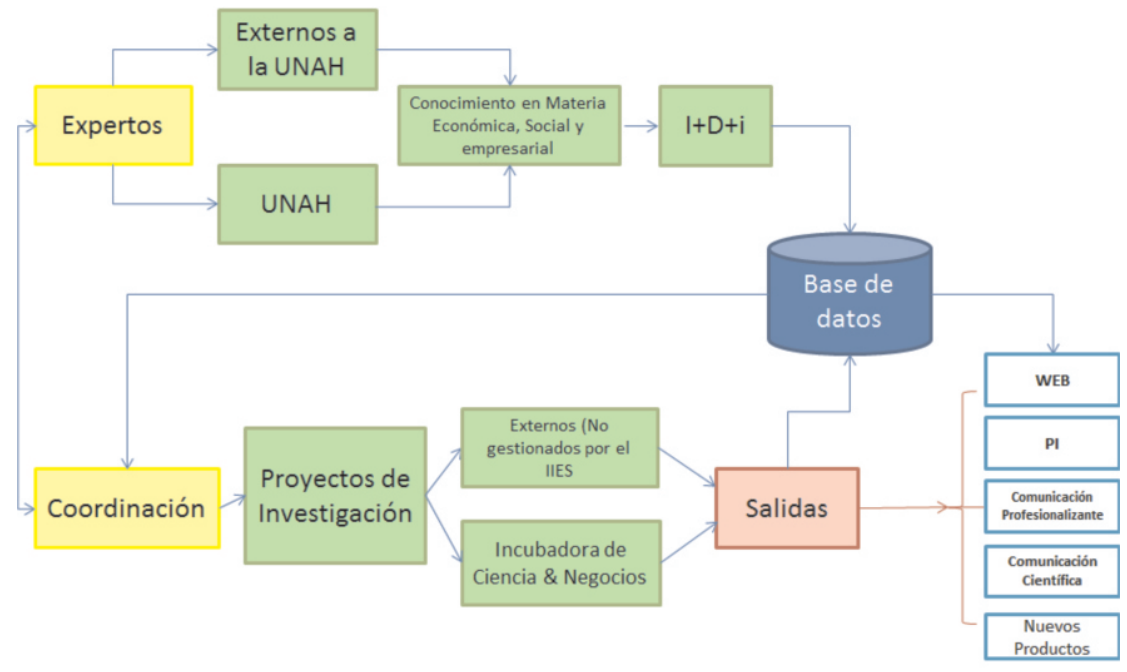

El observatorio Económico y de Emprendimiento de la UNAH estará asistido de forma directa por las incubadoras de Ciencia y Emprendimiento de forma directa, conjuntando sus bases de datos, para la utilización y transferencia de datos a la del Observatorio, dicho sistema se procura este modelado de la siguiente manera: La vinculación final y de forma integrada buscará realizar una base de datos en común, con subdivisiones que contemplen una base de datos del Observatorio Económico Empresarial. Las Incubadoras para tener una retroalimentación y dar soporte de forma conjunta al Observatorio, sistematizada tal acción bajo un concepto integrador de la siguiente forma:

Figura 4. Procesos del OEE Integrado, Fuente: IIES-UNAH 2011

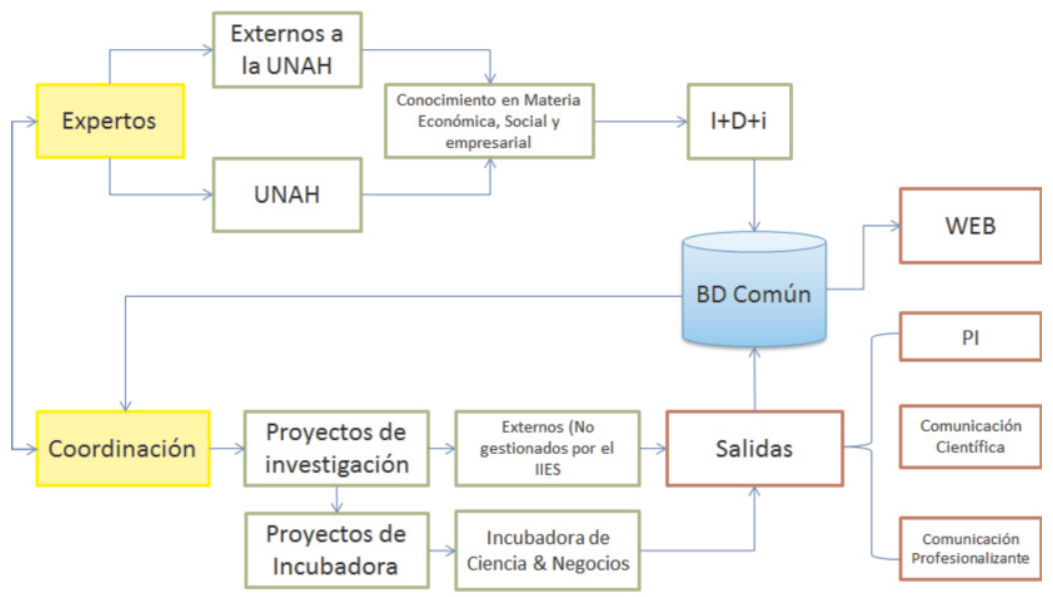


El proceso Integrado del Observatorio Económico y de Emprendimiento se fortalecerá con la reunión de expertos en temáticas diversas para debatir acerca de los resultados obtenidos en el Observatorio y dar soluciones estratégicas a corto plazo de carácter económico, político y social para beneficio de la sociedad.

\section{INDICADORES Y VARIABLES DE MEDICIÓN}

El observatorio Económico y de Emprendimiento tendrá como fin gestionar a través de sus expertos, estudiantes y personal docente investigador del IIES para alimentar las bases de datos de ocho divisiones macro y micro económicas creadas y con ello dar conocer datos de relevancia acerca del comportamiento de la economía local a fin de generar conocimiento y conciencia critica que de paso a la toma de decisiones estratégicas y a la generación de políticas macro y micro económicas. Acontinuación se enlistan las ocho divisiones:

1. Noticiario Económico: Será el espacio diseñado para la divulgación de los estudios e investigaciones realizadas por el personal docente investigador del Instituto de Investigaciones Económicas y Sociales de la UNAH, así como para todos los docentes, alumnos de pre y post grado, expertos y demás personas de la sociedad interesados en aportar datos para el Observatorio.

2. Economía: Dicho espacio será para la difusión de los indicadores macro económicos como ser; el IMAE, Reservas Internacionales, PIB, PNB, Tasas de interés, Subastas y Mercados de Divisas, Inversión Extranjera Directa, Inflación, Balanza de Pago, y cualquier otra variable macro o micro económica de relevancia que requiera su estudio desde la academia.

3. Empleo: En este apartado del Observatorio se estudiara los movimientos que ocurren de forma periódica e histórica, en el mercado laboral, empezando a nivel local y expandiéndonos a nivel nacional, regional e internacional.

4. Consumo: Debido a la inestabilidad económica que viven los países del mundo, en especial los de Centro América, es necesario involucrar a la academia en el estudio del comportamiento del consumidor, analizar las plazas, el índice de precios, los productos, la promoción, para con ello definir los productos de mayor consumo.

5. Emprendimiento: Este apartado pretende hacer una medición, a través del número de marcas y patentes, así como todos aquellos registros comerciales que surgen cada mes, de las actividades de emprendimiento originadas en el país. De igual forma se medirá el apoyo en materia de innovación, desarrollo, estrategias de mercado y competitividad, dado por el gobierno a este importante polo de desarrollo. 
6. Oferta y Demanda: El análisis de la oferta y demanda de la educación en Honduras dará paso a la medición de la calidad educativa y a reorientar la educación a las demandas que están presentes en los sectores económicamente activos.

7. Demográfico, Social: Los principales actores en los cambios económicos, son los mercados y los humanos, en tal sentido los nacimientos, defunciones, migraciones e emigraciones, resultan ser variables importantes y determinantes para el futuro económico de un país, por lo que su estudio es de carácter primordial.

8. Industria: La división industrial permitirá agrupar todos aquellos indicadores que faciliten la medición de la productividad en el país, a su vez, se mostrara de forma semanal el historial de cambios en los precios de los principales insumos de producción y cotizaciones de materias primas.

A continuación se enlistan los principales medios de verificación de la economía local analizados desde la academia, la cual será de uso para la toma de decisiones y aprendizaje de los estudiantes de pregrados y posgrado de la UNAH, en específico de la Facultad de Ciencias Económicas.

\section{Figura 5. Áreas de Análisis e Indicadores del OEE, Fuente: IIES-UNAH 2011}

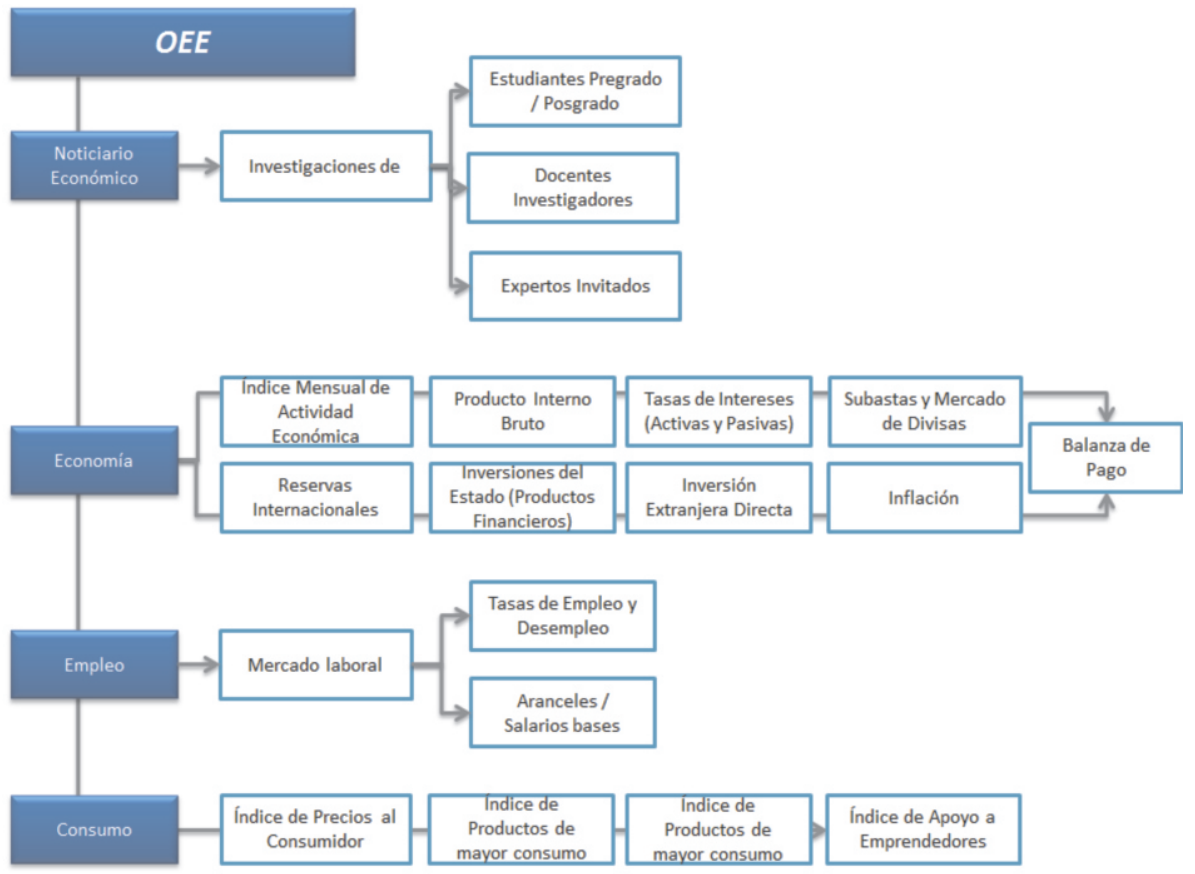



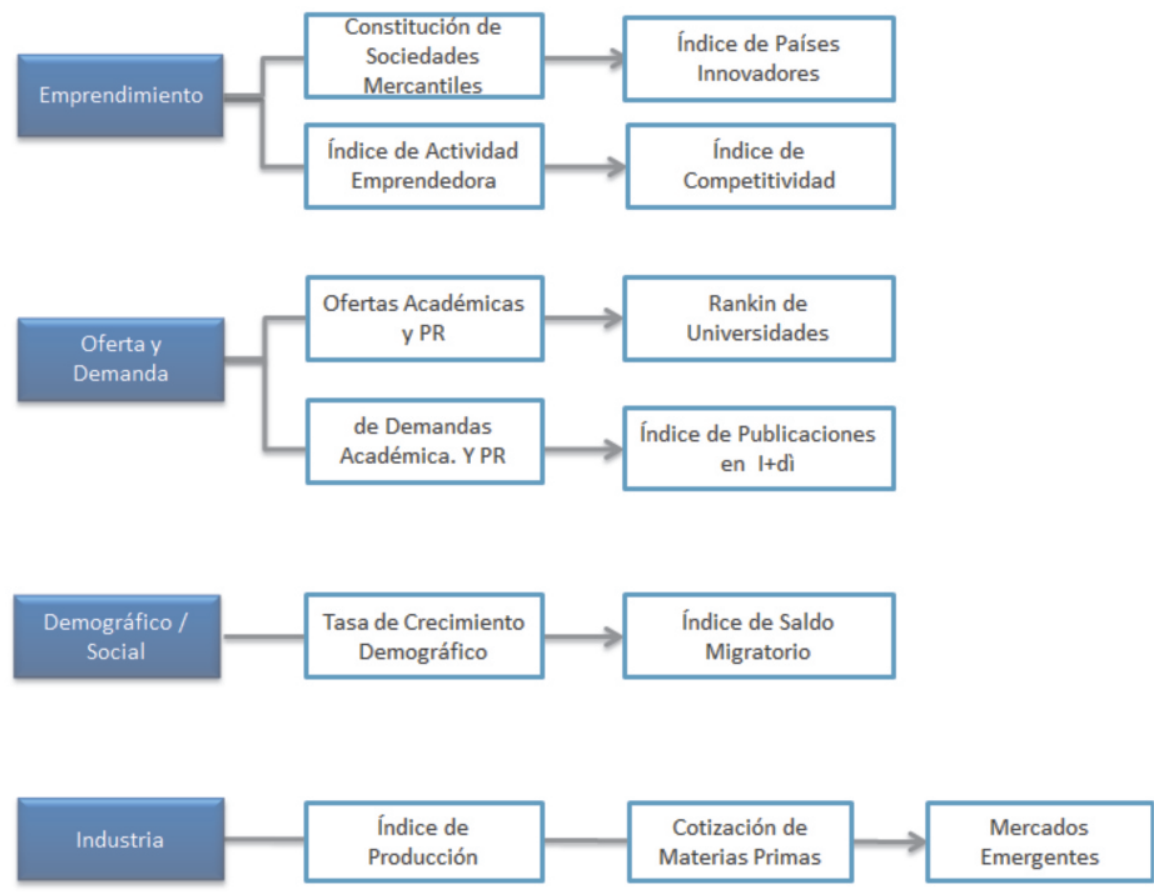

\section{BENEFICIOS DE LA IMPLEMENTACIÓN DE LOS OBSERVATORIOS DESDE LAACADEMIA}

La presente propuesta será la primera creación de un Observatorio Económico y de Emprendimiento en Honduras, abanderado por la Universidad Nacional Autónoma de Honduras, por lo tanto reviste un carácter de gran importancia e interés para académicos y profesionales a nivel nacional e internacional. Se espera que el desarrollo del mismo dé a conocer los movimientos de las economías locales y mundiales, así como el efecto que estas tendrían en las poblaciones, gestionando el desarrollo de investigaciones para crear medidas críticas preventivas que permitan la mejora en la competitividad en los sectores productivos del país.

Desde el punto de vista académico, será de beneficio para las carreras de la UNAH y en especial de la Faculta de Ciencias Económicas, ya que será un laboratorio para que estudiantes y profesores puedan interactuar y validar sus investigaciones con la realidad nacional y nutrir el proceso de enseñanza vinculando la formación teórica con la práctica. A su vez será un tanque de pensamientos sobre estudios económicos empresariales de amplio interés nacional. El OEE Sistematizará y creará índices de medición a través de los indicadores socioeconómicos y 
empresariales, brindando a los sectores interesados en conocer los movimientos constantes de la economía del país y el mundo, información valiosa y precisa a costos impensables, mediante un portal que les permitirá monitorear y ver el estado actual de nuestra economía.

\section{CONCLUSIONES}

La incursión de la academia en la creación de los Observatorios Económicos y de Emprendimiento dará lugar al fomento de la investigación científica desde los estudiantes hasta los catedráticos de la academia, al ser en primera instancia formadores de conciencia critica y en segunda instancia entes que permitan vislumbrar y adoptar medidas tanto preventivas como correctivas ante los problemas económicos que se pudieran dar a nivel nacional, regional y mundial.

La implementación del OEE permite la articulación de las diversas unidades de investigación de las academias y de igual forma contribuye a la sinergia entre las instituciones de formación y los entes reguladores y gobernantes de un país. Los resultados en temas estadísticos que presentan los observatorios permiten simular el comportamiento de la economía del país y con ello tomar acciones, por parte de los distintos sectores y a su vez por los miembros de los Think Tank, la cual darán como resultado estrategias de país, negocio y de economía individual que permitirán afrontar los nuevos retos en materia económica.

\section{AGRADECIMIENTOS}

Agradecemos infinitamente los esfuerzos que realiza la Dirección de Investigación Científica por promover, apoyar y financiar las actividades de investigación que de cada una de las unidades e institutos de la Universidad Nacional Autónoma de Honduras se derivan.

\section{REFERENCIAS}

Berdie, D. R., \&Anderson, J. F. (1986). Cuesitonarios; Diseño y Uso. Metuchen, New Jersey: Scarecrow Press, Inc.

Clark, C. (1971). Las condiciones del progreso económico . España: Editorial Alianza.

Drucker, P. (1985). Innovación y Emprendimiento. Colombia, Bogota: Editorial Norma. 
Fernández, R. (2006). Matemáticas Financieras y Evaluación de Proyectos. Administracion, Economia y Emprendimiento.

Heidi Blankenship, Viktor Kulhavý, Jonas Lagneryd. (2007). Introducing Strategic Sustainable Development in a business incubator. Karlskrona, Sweden: Blekinge Institute of Technology.

Hernández Sampieri, R. y. (1998). Metodología de la Investigación. D.F. Mexico: Editorial McGraw-Hill.

Kerlinger, F. N. (1983). Investigación del comportamiento, técnicas y métodos. DF. Mexico: Interamericana.

LIND, D. y. (2004). Estadística para administración y economia. Colombia: Alfaomega. 